\title{
Brain Processing of Fearful Facial Expression in Mentally Disordered Offenders
}

\author{
Katarina Howner ${ }^{1}$, Håkan Fischer ${ }^{2,3}$, Thomas Dierks ${ }^{4}$, Andrea Federspiel ${ }^{4}$, Lars-Olof Wahlund ${ }^{2}$, \\ Tomas Jonsson $^{5}$, Maria Kristoffersen Wiberg ${ }^{5}$, Marianne Kristiansson ${ }^{1}$ \\ ${ }^{1}$ Department of Clinical Neuroscience, Karolinska Institute, Stockholm, Sweden \\ ${ }^{2}$ NVS-Department, Karolinska Institute, Stockholm, Sweden \\ ${ }^{3}$ Department of Psychology, Stockholm University, Stockholm, Sweden \\ ${ }^{4}$ Department of Psychiatric Neurophysiology, University Hospital for Psychiatry, Bern, Switzerland \\ ${ }^{5}$ Department of Clinical Science, Intervention and Technology, Karolinska Institute, Stockholm, Sweden \\ E-mail: katarina.howner@ki.se \\ Received June 14, 2011; revised July 19. 2011; accepted July 28, 2011
}

\begin{abstract}
Emotional facial expressions are important cues for interaction between people. The aim of the present study was to investigate brain function when processing fearful facial expressions in offenders with two psychiatric disorders which include impaired emotional facial perception; autism spectrum disorder (ASD) and psychopathy (PSY). Fourteen offenders undergoing forensic psychiatric assessment (7 with ASD, and 7 psychopathic offenders) and 12 healthy controls (HC) viewed fearful and neutral faces while undergoing functional magnetic resonance imaging (fMRI). Brain activity (fearful versus neutral faces) was compared both between HC and offenders and between the two offender groups (PSY and ASD). Functional co-activation was also investigated. The offenders had increased activity bilaterally in amygdala and medial cingulate cortex as well as the left hippocampus during processing fearful facial expressions compared to HC. The two subgroups of offenders differed in five regions compared with each other. Results from functional co-activation analysis suggested a strong correlation between the amygdala and anterior cingulate cortex (ACC) in the left hemisphere only in the PSY group. These findings suggest enhanced neural processing of fearful faces in the amygdala as well as in other facial processing brain areas in offenders compared to HC. Moreover, the coactivation between amygdala and ACC in the PSY but not the ASD group suggested qualitative differences in amygdala activity in the two groups. Since the sample size is small the study should be regarded as a pilot study.
\end{abstract}

Keywords: Psychopathy, Autism Spectrum Disorder, Offenders, fMRI, Emotional Facial Processing

\section{Introduction}

Emotional facial expressions are unique cues, crucial for social interaction [1]. They convey information about how another individual feels, as well as the motivations and intentions of others. This increases our ability to predict the behavior of other persons and also possible threats, thus facilitating adjustment of our own behavior [2]. Impairment in perceiving emotional facial expressions can lead to strange and inappropriate behavior and may also lead to antisocial behavior. A meta analysis of antisocial populations and emotional facial recognition suggested a robust link between antisocial behavior and specific deficits in recognizing fearful expressions [3].

Psychopathy, as defined by Hare [4], and autism spectrum disorder (ASD) [5] are two developmental disorders with impairments in emotional facial expression processing [6-9]. Psychopathy has been associated with increased risk of offending behavior [10] and ASD has been suggested to be overrepresented in forensic psychiatric settings $[11,12]$.

ASD includes impairments in mentalizing, emotional reciprocity, communication, and social interaction [5]. Also impairment in perceiving emotional facial expressions has been suggested. Brain imaging studies have shown that subjects described as high-functional autism 
(HFA) have showed parallels to subjects with amygdala damage concerning facial perception $[9,13]$ and in other studies it have been showed that autistic subjects use alternative pathways in the brain when processing emotional facial expressions [14,15]. In comparison to healthy individuals who commonly focus on the eye-region when looking at human faces, these individuals seem to focus on other parts of the face, for example on the mouth [16]. Psychopathic subjects often present a good, though superficial, ability to understand social signals [17]. Despite this, it has been shown that these subjects often have specific impairments in recognizing sad and fearful facial expressions [18,19], as well as more global impairment in identifying facial expressions [6]. Regardless of the fact that both groups (ASD and PSY) demonstrate impairment in recognizing emotional facial expressions, the different clinical symptoms shown in these two groups could possible correspond to recruitment of different brain networks when processing various facial expressions. Blair has discussed Asperger syndrome and psychopathy in this respect and suggests that the amygdala could be the key structure here [20, 21].

A specific neural network for processing human faces has been suggested [22]. This network consists of brain regions processing static information, such as the shape and size of the face (including inferior occipital gyrus, lateral fusiform gyrus, and superior temporal sulcus), and areas specific for other aspects of facial perception. In this network, the amygdala has a specific role in processing emotional facial expression. It is not clarified whether there is differential neural processing of fearful faces in psychopathic offenders as compared to autistic offenders.

In the present functional magnetic resonance imaging (fMRI) study, we contrasted fearful and neutral faces [23] in a blocked design to study differences in cerebral activation between offenders with ASD or psychopathy and a healthy control group. In many previous studies on criminal offenders the inclusion criteria for subjects studied have varied substantially [24], so in the present study, we addressed this specific problem and were very restrictive in the inclusion criteria, in order to select well defined subjects, resulting in small but fairly homogeneous study groups. To the best of our knowledge, this is the first fMRI-study to compare offenders with ASD with psychopathic offenders in the forensic psychiatric setting, and the present study should be regarded as a pilot study.

We hypothesized that the offender group would exhibit a different pattern of neural activity in the network processing fearful facial expressions, compared to controls. We also hypothesized that the two subgroups of offenders would differ from each other in this network, especially in amygdala activation, and in functional coactivation between the amygdala and other regions within the network.

\section{Material and Methods}

\subsection{Subjects}

The sample consisted of 26 right-handed male subjects; 14 offenders ( 7 with ASD and 7 psychopathic offenders) undergoing forensic psychiatric assessment, and 12 healthy non-criminal controls. There were no age differences between the groups (Table 1).

Healthy controls (HC) were recruited from posters advertised at a nearby hospital. Offenders were recruited from the Department of Forensic Psychiatry in Stockholm. In Sweden major forensic psychiatric assessments are performed after the court has found evidence for the crime but before conviction. In this unit, approximately 280 forensic psychiatric assessments of inmates in custody are performed each year, $10 \%$ of inmates are female, and of the remaining 90\%, approximately 5\% have marked psychopathic traits (i.e. Psychopathy Check List Revised, PCL-R [4] score > 30) and a further $10 \%$ are diagnosed with autism spectrum disorder. The study subjects were recruited consecutively during, in all, 24 months.

Inclusion criteria for the offenders were either a main diagnosis of autism spectrum disorder (ASD) or psychopathy (PSY), as defined by Hare with PCL-R scores $>30$ [4]. Exclusion criteria were axis I diagnoses, difficulties in understanding Swedish or acute psychosis at the time of assessment.

All subjects underwent an interview including the Structured Clinical Interview for DSM-IV (SCID I) Screening Interview [25,26], Asperger Syndrome Diagnostic Interview, (ASDI) [27], and Psychopathy Check List Screening Version (PCL-SV) [28]. Age, intelligence quotient (IQ) according to the Wechsler Adult Intelligence Scale-Revised (WAIS-R) [29] and medication were collected from the forensic psychiatric reports (Table 1). After the MR-scans, all participants performed State Trait Anxiety Inventory State (STAI-S) [30] in order to measure how stressful the situation was perceived. One subject in the ASD group was HIV-positive without clinical symptoms, structural brain damage, and neuropsychological dysfunction. All analyses including the ASD group were performed both with and without this subject, and the results remained stable.

The study was approved and conducted in accordance with ethical guidelines established by the Regional Ethical Committee at the Karolinska Institutet in Stockholm. 
Table 1. Demographic and clinical characteristics. Mean value, SD in parenthesis. Mann-Whitney U-test was used in comparisons between groups.

\begin{tabular}{ccccc}
\hline & HC & Offenders & ASD & PSY \\
\hline Age & $28.1(8.2)$ & $28.6(6.9)$ & $29.9(4.9)$ & $27.3(4.9)$ \\
IQ & - & $98.1(17.7)$ & $100(22.3)$ & $94.8(4.3)$ \\
PCL-SV* & $0.5(0.5)$ & $15.1(5.5)$ & $10.6(3.5)$ & $19.7(2.1)$ \\
STAI-S & $32.6(11.6)$ & $42.4(16.4)$ & $41.5(19.1)$ & $43.1(15.4)$ \\
\hline
\end{tabular}

*Significant differences in HC vs Offenders: $p<0.0001$, and PSY vs ASD: $p<0.0001$; HC = healthy controls; ASD = offenders with autism spectrum disorder; $\mathrm{PSY}=$ psychopathic offenders; IQ = intelligence quotient; PCL-SV = Psychopathy Checklist-Screening Version; STAI-S = State Trait Anxiety Inventory-State.

After description of the study, written informed consent was obtained.

\subsection{Statistical Analysis of Demographic Variables}

Age, IQ, PCL-SV scores, and STAI-S scores are presented as means (SD) and tested with Mann Whitney U-test. The confidence interval was set at $95 \%$, and the level of statistical significance of differences was $p<$ 0.05 .

\subsection{PCL-SV Scoring}

The PCL-SV was performed in all study subjects in order to make sure that no subjects in the ASD and the HC group had a high amount of psychopathic traits. The PCL-SV was used as the HC group consisted of non criminals. It has high correlation with the PCL-R [31,32] but is preferred for use in non-criminal settings. The PCL-SV rating was assessed by two independent raters and the cut off score for psychopathy in the PCL-SV was 18.

Inter-rater reliability was computed using the intraclass correlation coefficient (ICC) [33], which was calculated using a two-way mixed effects model. The single measure ICC was $0.98(95 \% \mathrm{CI}=0.95-0.99, n=25)$.

Two-tailed $t$-tests showed significant differences between the HC group and the offenders $(t=9.93, \mathrm{df}=$ $13.3, p<0.0001)$. The same analysis was performed between the two subgroups of offenders (PSY vs AUT) ( $t=$ 5.95, $\mathrm{df}=9.7, p<0.0001)$. For mean scores see Table 1.

\subsection{Stimuli}

The stimuli comprised photographs of human facial expressions from the standardized Ekman and Friesen face set [23]. A blocked design was used which consisted of alternating blocks of fearful and neutral faces. Each block consisted of 15 different faces, presented for 2 seconds each, followed by a fixation cross for $400 \mathrm{~ms}$.

The $36 \mathrm{sec}$ 'face-blocks' were interspersed with $18 \mathrm{sec}$ "rest-blocks" with a white fixation cross on a black screen. The subjects were asked to identify the sex (male or female) of the face by pressing buttons with the right index finger. The pictures were presented with a projector on a screen and the subjects viewed the pictures through a mirror on the head coil.

\subsection{Behavioral Data}

Mean reaction time and accuracy according to the sex discrimination task were used as a proxy for attention to the faces. Data are presented as means (SD) and comparisons between groups were performed using twotailed $t$-test. Behavioral data was missing for one subject in the ASD group due to technical problems.

\subsection{MRI Acquisition}

The MR-scans were acquired with Siemens Avanto $1.5 \mathrm{~T}$ whole body MRI system, with a 12-channel matrix head coil. The subjects were placed supine in the scanner wearing headphones to reduce noise from the machine, and their heads were fixated with a vacuum pillow.

Functional imaging was performed using a T2*weighted gradient echo planar imaging sequence (EPI)mosaic sequence $(\mathrm{TR}=3000 \mathrm{~ms}$, $\mathrm{TE}=50 \mathrm{~ms}$, slice thickness $=5 \mathrm{~mm}$, gap between slices $=0.5 \mathrm{~mm}, \mathrm{FOV}=$ $220 \mathrm{~mm}$, matrix size $=64 \times 64$, voxel dimension $=3.4$ $\mathrm{mm} \times 3.4 \mathrm{~mm} \times 5 \mathrm{~mm}, 30$ coronal slices, covering the whole brain), 114 volumes were collected. For structural data we used a 3D magnetization-prepared rapid-acquisition gradient echo (MP-RAGE) sequence, 176 sagittal slices were acquired with the following parameters: repetition time $(\mathrm{TR})=2300 \mathrm{~ms}$, inversion time $(\mathrm{TI})=$ $1100 \mathrm{~ms}$, echo time $(\mathrm{TE})=3.93 \mathrm{~ms}$, slice thickness $=1$ $\mathrm{mm}$, field of view $(\mathrm{FOV})=256 \mathrm{~mm} \times 256 \mathrm{~mm}$, Matrix $=$ 
$256 \times 256$, isotropic voxel size $=1 \mathrm{~mm}^{3}$.

\section{7. fMRI-Analyses}

The first three volumes in each run were discarded to allow for $\mathrm{T} 1$ equilibration effects. Image time-series analysis was performed using Brain voyager, BVQX 1.9. Preprocessing comprised a correction of slice scan time, 3D motion correction (Trilinear/sinc interpolation) and temporal filtering removing linear trend. The images of each subject were co registered to the $3 \mathrm{D}$ anatomical volume, normalized into Talairach space, and herby resample into $3 \mathrm{~mm}$ isotropic voxels. The resulting volumetime course files (VTC) were then spatially smoothed with a Gaussian filter of $8 \mathrm{~mm}$ full-width at half maximum for the group analyzes.

We chose the following anatomical region of interests (ROIs), from the model for facial perception by Haxby et al. [22]; inferior occipital gyrus, superior temporal sulcus, lateral fusiformis gyrus, insula, hippocampus, parahippocampus, cingulate gyrus and amygdala. Within these predefined areas, we used a threshold level of $p<0.001$ (uncorrected) in line with many other studies in the field. Because the amygdala has been shown in many studies to be of major importance for fearful facial processing [34], we used a threshold of $p<0.05$ (uncorrected) in this specific ROI. We also performed a whole brain analysis, in which we used a threshold level of $p<0.05$ (FDRcorrected).

Individual analysis; we investigated blood oxygen level dependent (BOLD) activation associated specifically with the processing of the emotional cues in fearful faces (i.e fearful faces $>$ neutral faces). This was done by applying a fixed effects model [35], with condition-specific stimulus boxcar functions, convolved with a gammakernel to model the hemodynamic response behaviour. Three predictors were entered into the design matrix: fear faces, neutral faces, and baseline (looking at a white fixation cross on a black screen). The outputs of the model are beta values for the different conditions.

Between-group analyses were performed between (1) the HC and the offenders and (2) between the two subgroups of offenders (ASD vs PSY). Random effects models were used for both within and between group analyses. The between group analyses were calculated in the fo- llowing steps: first all individual VTCs were created for the contrast fearful faces $>$ neutral faces, then a twotailed $t$-test was performed between the HC group and the offender group and finally the same procedure was performed between the ASD and the PSY groups.

\subsection{Functional Co-Activation}

The functional co-activation analysis was performed in the following steps: first functional ROIs in the right and the left amygdala in the offender group were defined based on the group contrast fearful faces $>$ neutral faces, consisting of 8 voxles located around the peak activation voxel in each of the two regions (mean TAL: $X=21, Y$ $=-11, Z=-11 ; X=-19, Y=-7, Z=-13)$.

Individual beta values from these specific ROIs were collected. The difference between the beta values for the fear condition minus the beta value for the neutral condition for each subject was used as a measurement of amygdala reactivity to fearful expressions. From the between group analyses (ASD vs PSY), we found five regions (Table 3) where the two offender groups differed from each other within the network for the processing facial expressions [22]. In all these five regions we defined functional ROIs, as described above for the amygdala, and extracted beta values from these ROIs as well. We then correlated amygdala reactivity with activity in these specific ROIs, using Pearsons correlation, on each side separately (right and left), within each of the two groups (ASD and PSY). Grubb's test was used to test for outliers [36].

\section{Results}

\subsection{Behavioral Responses}

There were no significant differences between groups in any of the behavioral responses (Table 2).

\section{2. fMRI BOLD-Responses}

\subsubsection{Between-Group Comparisons of Fearful Versus Neutral Faces Contrast}

The offender group, collapsed across diagnoses, compared to $\mathrm{HC}$ had higher activation in the amygdala bilat-

Table 2. Behavioral responses. Mean values and SD in parenthesis.

\begin{tabular}{|c|c|c|c|c|}
\hline & HC & Offenders & ASD & PSY \\
\hline Accuracy (\%) & $96.9(2.9)$ & $96.3(3.4)$ & $95.7(3.7)$ & $96.9(3.3)$ \\
\hline Reaction time, neutral (ms) & $668.2(89.7)$ & $725.6(97.9)$ & $759.2(130.6)$ & $696.8(53.5)$ \\
\hline Reaction time, fear (ms) & $671.7(111.1)$ & $686.5(74.9)$ & $703.7(98.8)$ & $671.8(50.3)$ \\
\hline
\end{tabular}

$\mathrm{HC}=$ healthy controls; ASD $=$ offenders with autism spectrum disorder; PSY = psychopathic offenders 
Table 3. Between-group comparisons. The contrast fear $>$ neutral, $p<0.001$ (uncorrected).

\begin{tabular}{|c|c|c|c|c|c|c|c|}
\hline Region & Brodmann's Area & Hemisphere & $\mathrm{X}$ & Y & Z & Numbers of voxels & $t$-value \\
\hline \multicolumn{8}{|c|}{ Offenders $>\mathrm{HC}$} \\
\hline Amygdala & Amygdala & $\mathrm{L}$ & -19 & -7 & -13 & 118 & 2.22 \\
\hline Amygdala & Amygdala & $\mathrm{R}$ & 21 & -11 & -11 & 126 & 2.18 \\
\hline Cingulum & 24 & $\mathrm{R}$ & 21 & 0 & 34 & 7 & 3.85 \\
\hline Cingulate gyrus & 23 & $\mathrm{~L}$ & -23 & 23 & 27 & 44 & 3.93 \\
\hline Parahippocampus-Hippocampus & Hippocampus & $\mathrm{L}$ & -43 & -33 & -5 & 22 & 3.89 \\
\hline \multicolumn{8}{|c|}{$\mathrm{HC}>$ Offenders } \\
\hline \multicolumn{8}{|l|}{ No areas } \\
\hline \multicolumn{8}{|c|}{ PSY $>$ ASD } \\
\hline Insula & 13 & $\mathrm{~L}$ & -39 & -7 & -5 & 24 & 4.36 \\
\hline Anterior cingulate cortex & 24 & $\mathrm{~L}$ & -4 & 27 & 17 & 19 & 4.40 \\
\hline \multicolumn{8}{|c|}{ ASD $>$ PSY } \\
\hline Insula & 13 & $\mathrm{R}$ & 34 & -25 & 0 & 258 & 4.61 \\
\hline Lingual gyrus/fusiform gyrus & 18 & $\mathrm{~L}$ & -7 & -91 & -14 & 26 & 4.37 \\
\hline Cingulate gyrus & 23 & $\mathrm{~L}$ & -15 & -13 & 31 & 7 & 4.45 \\
\hline
\end{tabular}

$\mathrm{HC}=$ healthy controls; PSY = psychopathic offenders; $\mathrm{ASD}=$ offenders with autism spectrum disorder.

erally, the left hippocampus as well as in the medial cingulate cortex bilaterally (Table 3 ).

In a comparison between the two subgroups of offenders (ASD vs PSY), there were two regions where the PSY group had significantly higher activation than the ASD group; anterior cingulate cortex (ACC), and insula on the left side (Table 3, Figure 1). The ASD group, on the other hand, had higher activation in the right insula and left cingulate cortex, and left fusiform gyrus (Table 3). In the whole brain analysis we did not find any significant differences in any of the between group analyses.

\subsubsection{Functional Co-Activation}

In the PSY group there was a correlation between the amygdala and the ACC on the left side $\left(r^{x y}=0.97, p<\right.$ $0.001)$, which was not found in the ASD group $\left(r^{x y}=\right.$ $0.327, p=0.475$ ) (Figure 2). No other correlations with amygdala reactivity were significant in any of the two groups.

\section{Discussion}

The present study investigated the neural underpinnings of emotional facial perception in offenders with ASD or psychopathy. The whole offender group had increased BOLD activity, compared to the $\mathrm{HC}$ group, in specific nodes in the neural network involved in perceiving and processing facial information [34] namely bilateral amygdala, cingulate gyrus, and left hippocampus. In studies applying brain imaging techniques to other groups of antisocial subjects, functional impairments in the frontal lobes [37] and the limbic system [38,39] have been demonstrated, and a suggested impairment in the balance between these two systems has been described [40-42]. These studies have reported both hypoactivity $[38,43]$ and hyperactivity $[40,42]$ in the amygdala and the limbic system compared to healthy controls.

Hyper activation in amygdala in the current offender group could reflect an imbalance between the limbic system and more frontal systems located outside of the network processing facial expressions; it could also be related to alteration in the way fearful facial expressions are processed in these specific groups. Since both of these groups (PSY and ASD) have shown impairments in recognizing fearful facial expressions before [3,6,9,44], the enhanced amygdala activity may be related to ambiguity in the processing of the emotional face signal [45]. If so, this ambiguity could be related to enhance cognitive elaborative processing associated with exposure to fearful faces in the offender group, which could explain the activations also in more "cognitive regions", such as the medial cingulate gyrus and the hippocampus.

In the contrast between the two offender groups (ASD 

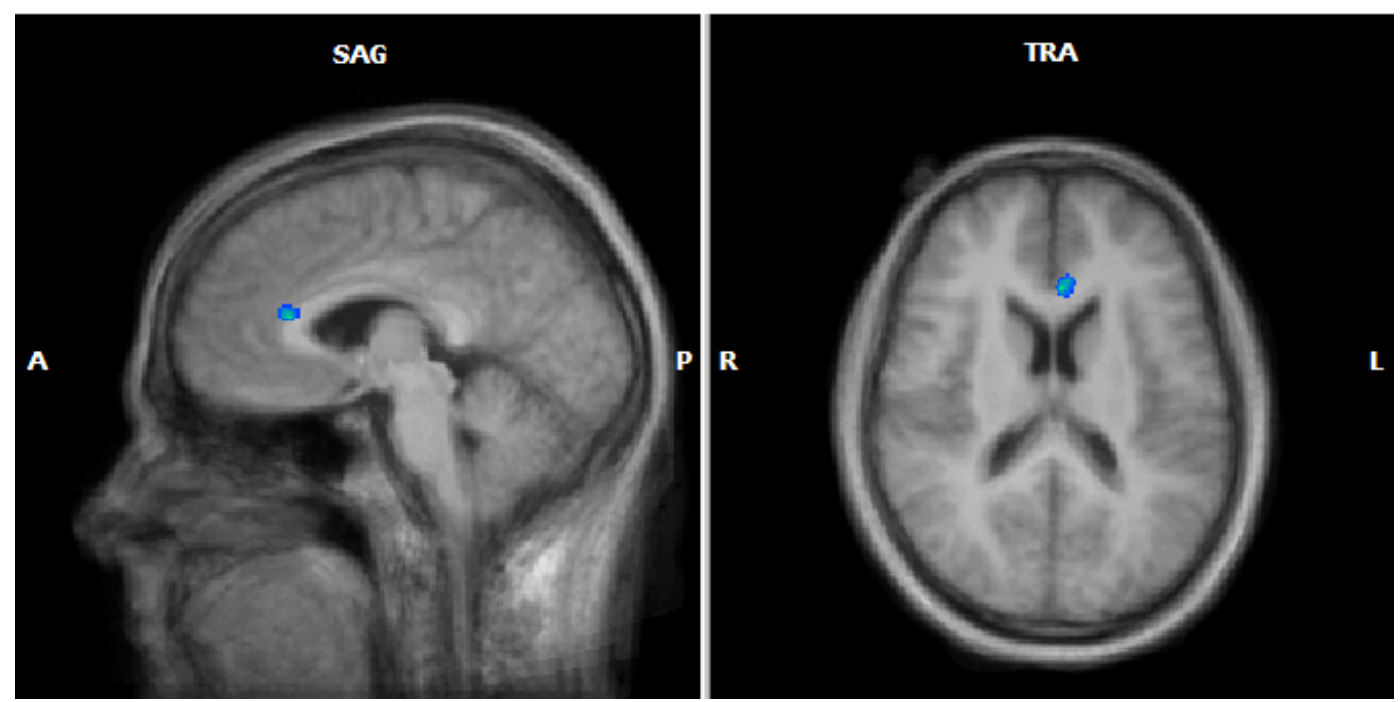

Figure 1. Between-group analyses. Blue dot illustrates PSY vs ASD in the contrast fearful > neutral faces; PSY > ASD in ACC (mean TAL-coordinates: $-4,27,17), p$-value $<0.01$ (corrected). This region was correlated with amygdala in the PSY-group. PSY = psychopathic offenders; ASD = offenders with autism spectrum disorder syndrome; ACC = anterior cingulate cortex.

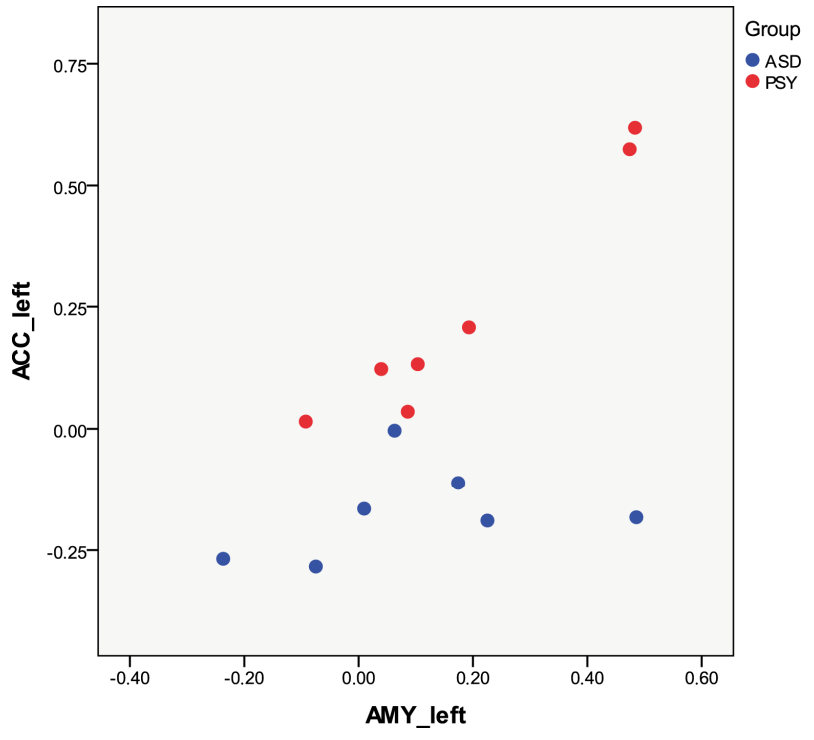

Figure 2. Functional co-activation. Correlation between reactivity in the amygdala (AMY) and in the anterior cingulate cortex (ACC), on the left side in the group of psychopathic offenders, PSY, $\left(r^{x y}=0.97, p<0.001\right)$, but no significant correlation in the group of offenders with autism spectrum disorder, ASD, $\left(r^{x y}=0.327, p=0.475\right)$.

vs PSY) we observed differential activations in both the insular cortex (left vs right) and the cingulate gyrus. Hence, ASD and PSY seem to have differential activation patterns within these two cortical regions during perception of fearful faces. The insula cortex has been connected to visceral representation of autonomic arousal $[46,47]$. Because the ASD-group activated the right insula more than the PSY-group this could reflect that exposure to fearful facial expressions is associated with more bodily arousal in the ASD-group compared to the PSY-group. This reasoning is in line with earlier studies showing that psychopathic subjects have lower autonomic responses during exposure to emotionally relevant information [43,48-52]. The PSY-group had higher activation in the ACC compared to the ASD- group which could be connected to the possible inhibition of amygdala reactivity. The ASD-group, on the other hand, had higher activity in the posterior cingulated gyrus, Brodmann area 23. The posterior cingulated cortex has been connected to both emotional processing and memory-related functions [53-55]. Accordingly, the higher posterior cingulate activation in the ASD-group could reflect altered emotional or memory related processing of fearful facial information compared to the PSY-group.

Our third main result is a strong correlation between amygdala reactivity and ACC activity in the PSY group on the left side, which was not found in the ASD group. This suggests that the amygdala activation in the two offender groups may differ qualitatively, even though there were no quantitative differences. The ACC influences emotional processing [56] and is suggested a role in modulating the amygdala [57]. A possible explanation in the current study is that the ACC in the PSY-group inhibits the amygdala reaction, resulting in impaired emotional information processing. Hence, the amygdalaACC correlation could reflect a marked ACC influence on fearful emotional facial processing in the amygdala resulting in the disturbed fearful facial processing pattern often seen in psychopathic subjects [6,7]. An important note is that it is not possible to tell anything about the 
direction of the co-activation with the current analysis.

Some limitations have to be mentioned. Firstly, the sample size in the subgroups was small, thus increasing the risk of type II error, and the present study should be regarded as a pilot study. Secondly, five of the offenders and one $\mathrm{HC}$ were on medication. It was not possible to withdraw all medication, as the study was conducted in a clinical context. Some offenders had a history of drug and alcohol misuse prior to arrest; as they were remanded in custody, all of the offenders had been free from drugs and alcohol for at least 6 - 10 weeks prior inclusion. Finally, the use of $\mathrm{HC}$ as a comparison group to the offenders involves some problems; HC consisted of non criminals who probably have different socioeconomic status than the offenders, they all had completed high school education and were not tested with WAIS-R.

In summary, our findings indicate altered neural processing of fearful facial expressions in the offender group compared to $\mathrm{HC}$ within the neural network involved in processing facial information [22]. Moreover, the two subgroups of offenders differed from each other in direct or indirect functional communication between the amygdala and ACC, both located within the face processing network. The behavioral relevance of these differences is however unclear. Facial processing most certainly interacts with our decision making in various situations and is one important factor in the development of empathy [58]. Lack of empathy is a common trait in offenders [58]. Both psychopathy [4] and ASD [5] have been associated with reduced empathic ability. Empathy is a construct consisting of many aspects; for example emotional and cognitive empathy. While cognitive empathy or theory of mind requires mentalizing, emotional empathy includes the ability to interpret emotional facial expressions. In ASD the cognitive empathy is affected, which have not yet been found in psychopathy. Blair discussed these two disorders and their differences regarding the empathic ability. He points out their different dysfunctions, both regarding empathy deficit and differences in amygdala, and suggests that a "fine cut" between autism and psychopathy can be made in both amygdala and in the different aspects of empathy, emotional and cognitive empathy $[20,21]$. In future studies, brain activation patterns to facial expressions in subjects with autistic and psychopathic traits, with and without offending behavior, ought to be compared and related to measures of empathy and its different aspects.

\section{Acknowledgements}

We are grateful to Kristina Sygel for language revision and Kerstin Eriksson for assisting during the MRI-scans. This research has made use of the SMILE medical im- aging laboratory at Karolinska University Hospital, Stockholm. Financial support was provided through the regional agreement on medical training and clinical research between Stockholm County Council and the Karolinska Institutet (ALF) and grants from the National Board of Forensic Medicine in Sweden. Dr Fischer was funded by grants from the Swedish Research Council.

\section{References}

[1] P. Ekman, E. R. Sorenson and W. V. Friesen, "Pan-Cultural Elements in Facial Displays of Emotion," Science, Vol. 164, No. 875, 1969, pp. 86-88. doi:10.1126/science.164.3875.86

[2] R. J. Blair, "Facial Expressions, Their Communicatory Functions and Neuro-Cognitive Substrates," Philosophical Transactions of the Royal Society B: Biplogical Sciences, Vol. 358, No. 1431, 2003, pp. 561-572. doi:10.1098/rstb.2002.1220

[3] A. A. Marsh and R. Blair, "Deficits in Facial Affect Recognition among Antisocial Populations: A Meta-Analysis," Neuroscience \& Biobehavioral Reviews, Vol. 32, No. 3, 2008, pp. 454-465.

[4] R. D. Hare, "Manual for the Revised Psychopathy Checklist," Multi-Health Systems, Toronto, 2003.

[5] American Psychiatric Association, "Diagnostic and Statistical Manual of Mental Disorders-Text Revision," APA, Washington, DC, 2000.

[6] M. E. Hastings, J. P. Tangney and J. Stuewig, "Psychopathy and Identification of Facial Expressions of Emotion," Personality and Individual Differences, Vol. 44, No. 7, 2008, pp. 1474-1483. doi:10.1016/j.paid.2008.01.004

[7] Q. Deeley, et al., "Facial Emotion Processing in Criminal Psychopathy, Preliminary Functional Magnetic Resonance Imaging Study," The British Journal of Psychiatry, Vol. 189, 2006, pp. 533-539. doi:10.1192/bjp.bp.106.021410

[8] H. D. Critchley, et al., "The Functional Neuroanatomy of Social Behaviour: Changes in Cerebral Blood Flow When People with Autistic Disorder Process Facial Expressions," Brain, Vol. 123, No. 11, 2000, pp. 2203-2212. doi:10.1093/brain/123.11.2203

[9] R. Adolphs, L. Sears and J. Piven, "Abnormal Processing of Social Information from Faces in Autism," Journal of Cognitive Neuroscience, Vol. 13, No. 2, 2001, pp. 232240. doi:10.1162/089892901564289

[10] M. Dolan and M. Doyle, "Violence Risk Prediction. Clinical and Actuarial Measures and the Role of the Psychopathy Checklist," The British Journal of Psychiatry, Vol. 177, 2000, pp. 303-311. doi:10.1192/bjp.177.4.303

[11] P. Scragg and A. Shah, "Prevalence of Asperger's Syndrome in a Secure Hospital," The British Journal of Psychiatry, Vol. 165, No. 5, 1994, pp. 679-682. doi:10.1192/bjp.165.5.679

[12] M. Kristiansson and K. Sorman, "Autism Spectrum Disorders: Legal and Forensic Psychiatric Aspects and Reflections," Clinical Neuropsychiatry: Journal of Treat- 
ment Evaluation, Vol. 5, No. 1, 2008, pp. 55-61.

[13] M. A. Howard, et al., "Convergent Neuroanatomical and Behavioural Evidence of an Amygdala Hypothesis of Autism," NeuroReport, Vol. 11, No. 13, 2000, pp. 29312935. doi:10.1097/00001756-200009110-00020

[14] D. Hubl, et al., "Functional Imbalance of Visual Pathways Indicates Alternative Face Processing Strategies in Autism," Neurology, Vol. 61, No. 9, 2003, pp. 12321237.

[15] R. T. Schultz, et al., "Abnormal Ventral Temporal Cortical Activity during Face Discrimination among Individuals with Autism and Asperger Syndrome," Archives of General Psychiatry, Vol. 57, No. 4, 2000, pp. 331-340. doi:10.1001/archpsyc.57.4.331

[16] A. Klin, W. Jones, R. Schultz, F. Volkmar and D. Cohen, "Visual Fixation Patterns during Viewing of Naturalistic Social Situations as Predictors of social Competence in individuals with Autism," Archives of General Psychiatry, Vol. 59, No. 9, 2002, pp. 809-816. doi:10.1001/archpsyc.59.9.809

[17] M. Dolan and R. Fullam, "Theory of Mind and Mentalizing Ability in antisocial Personality Disorders with and without Psychopathy," Psychological Medicine, Vol. 34, No. 6, 2004, pp. 1093-1102. doi:10.1017/S0033291704002028

[18] R. J. R. Blair, et al., "Reduced Sensitivity to Others' Fearful Expressions in Psychopathic Individuals," Personality and Individual Differences, Vol. 37, No. 6, 2004, pp. 1111-1122. doi:10.1016/j.paid.2003.10.008

[19] R. J. Blair, E. Colledge, L. Murray and D. G. Mitchell, "A Selective Impairment in the Processing of Sad and Fearful Expressions in Children with Psychopathic Tendencies," Journal of Abnormal Child Psychology, Vol. 29, No. 6, 2001, pp. 491-498. doi:10.1023/A:1012225108281

[20] R. Blair, "Fine Cuts of Empathy and the Amygdala: Dissociable Deficits in Psychopathy and Autism," The Quarterly Journal of Experimental Psychology, Vol. 61, No. 1, 2008, pp. 157-170.

[21] R. J. Blair, "Responding to the Emotions of Others: Dissociating Forms of Empathy through the Study of Typical and Psychiatric Populations," Consciousness and Cognition, Vol. 14, No. 4, 2005, pp. 698-718. doi:10.1016/j.concog.2005.06.004

[22] J. V. Haxby, E. A. Hoffman and M. I. Gobbini, "Human Neural Systems for Face Recognition and Social Communication," Biological Psychiatry, Vol. 51, No. 1, 2002, pp. 59-67. doi:10.1016/S0006-3223(01)01330-0

[23] P. Ekman and W. Friesen, "Pictures of Facial Affect," Consulting Psychologist Press, Inc., Palo Alto, 1976.

[24] K. Wahlund and M. Kristiansson, "Aggression, Psychopathy and Brain Imaging-Review and Future Recommendations," International Journal of Law and Psychiatry, Vol. 32, No. 4, 2009, pp. 266-271.

[25] M. B. First, R. L. Spitzer, M. Gibbon and J. B. W. Williams, "Structured Clinical Interview for DSM-IV Axis I Disorders. Clinical Version. Administration Booklet," American Psychiatric Press, Washington DC, 1997.
[26] M. B. First, R. L. Spitzer, M. Gibbon and J. B. W. Williams, "Structured Clinical Interview for DSM-IV Axis I Disorders-Non-patient Edition," Biometric Research, New York State Psychiatric Institute, New York, 1997.

[27] C. Gillberg, M. Rastam and E. Wentz, "The Asperger Syndrome (and High-Functioning Autism) Diagnostic Interview (ASDI): A Preliminary Study of a New Structured Clinical Interview," Autism, Vol. 5, No. 1, 2001, pp. 57 66. doi:10.1177/1362361301005001006

[28] S. D. Hart, D. N. Cox and R. D. Hare, "Manual for the Psychopathy Checklist: Screening Version (PCL:SV)," Multi-Health Systems, Toronto, 1995.

[29] D. Wechsler, "WAIS-R Manual," The Psychological Corporation, New York, 1981.

[30] C. D. Spielberger, R. L. Gorsuch, R. Lushene, P. R. Vagg and J. A. Jacobs, "Manual for the State-Trait Anxiety Inventory," Consulting Psychological Press, Palo Alto, 1983.

[31] L. S. Guy and K. S. Douglas, "Examining the Utility of the PCL:SV as a Screening Measure Using Competing Factor Models of Psychopathy," Psychological Assessment, Vol. 18, No. 2, 2006, pp. 225-230. doi:10.1037/1040-3590.18.2.225

[32] D. J. Cooke, C. Michie, S. D. Hart and R. D. Hare, "Evaluating the Screening Version of the Hare Psychopathy Checklist-Revised (PCL:SV): An Item Response Theory Analysis," Psychological Assessment, Vol. 11, No. 1, 1999, pp. 3-13. doi:10.1037/1040-3590.11.1.3

[33] P. E. Shrout and J. L. Fleiss, "Intraclass Correlations: Uses in Assessing Rater Reliability," Psychological Bulletin, Vol. 86, No. 2, 1979, pp. 420-428. doi:10.1037/0033-2909.86.2.420

[34] F. C. Murphy, I. Nimmo-Smith and A. D. Lawrence, "Functional Neuroanatomy of Emotions: A Meta-analysis," Cognitive, Affective, \& Behavioral Neuroscience, Vol. 3, No. 3, 2003, pp. 207-233. doi:10.3758/CABN.3.3.207

[35] K. J. Friston, et al., "Analysis of fMRI Time-Series Revisited," NeuroImage, Vol. 2, No. 1, 1995, pp. 45-53. doi:10.1006/nimg.1995.1007

[36] NIST/SEMATECH, "E-Handbook of Statistical Methods," http://www.itl.nist.gov/div898/handbook/

[37] A. Raine, T. Lencz, S. Bihrle, L. LaCasse and P. Colletti, "Reduced Prefrontal Gray Matter Volume and Reduced Autonomic Activity in Antisocial Personality Disorder,' Archives of General Psychiatry, Vol. 57, No. 2, 2000, pp. 119-127.

[38] K. A. Kiehl, et al., "Limbic Abnormalities in Affective Processing by Criminal Psychopaths as Revealed by Functional Magnetic Resonance Imaging," Biological Psychiatry, Vol. 50, No. 9, 2001, pp. 677-684. doi:10.1016/S0006-3223(01)01222-7

[39] M. P. Laakso, et al., "Psychopathy and the Posterior Hippocampus," Behavioural Brain Research, Vol. 118, No. 2, 2001, pp. 187-193. doi:10.1016/S0166-4328(00)00324-7

[40] F. Schneider, et al., "Functional Imaging of Conditioned Aversive Emotional Responses in Antisocial Personality 
Disorder," Neuropsychobiology, Vol. 42, No. 4, 2000, pp. 192-201. doi:10.1159/000026693

[41] A. Raine, et al., "Reduced Prefrontal and Increased Subcortical Brain Functioning Assessed Using Positron Emission Tomography in Predatory and Affective Murderers," Behavioral Sciences \& the Law, Vol. 16, No. 3, 1998, pp. 319-332. doi:10.1002/(SICI)1099-0798(199822)16:3<319::AID-B $\underline{\mathrm{SL} 311>3.0 . \mathrm{CO} ; 2-\mathrm{G}}$

[42] J. L. Muller, et al., "Abnormalities in Emotion Processing within Cortical and Subcortical Regions in Criminal Psychopaths: Evidence from a Functional Magnetic Resonance Imaging Study Using Pictures with Emotional Content," Biological Psychiatry, Vol. 54, No. 2, 2003, pp. 152-162. doi:10.1016/S0006-3223(02)01749-3

[43] N. Birbaumer, et al., "Deficient Fear Conditioning in Psychopathy: A Functional Magnetic Resonance Imaging Study," Archives of General Psychiatry, Vol. 62, No. 7, 2005, pp. 799-805. doi:10.1001/archpsyc.62.7.799

[44] D. S. Kosson, Y. Suchy, A. R. Mayer and J. Libby, "Facial Affect Recognition in Criminal Psychopaths," Emotion, Vol. 2, No. 4, 2002, pp. 398-411. doi:10.1037/1528-3542.2.4.398

[45] M. Davis and P. J. Whalen, "The Amygdala: Vigilance and Emotion," Molecular Psychiatry, Vol. 6, No. 1, 2001, pp. 13-34. doi:10.1038/sj.mp.4000812

[46] A. D. Craig, "How do You Feel? Interoception: The Sense of the Physiological Condition of the Body," Nature Reviews Neuroscience, Vol. 3, No. 8, 2002, pp. 655666.

[47] C. B. Saper, "The Central Autonomic Nervous System: Conscious Visceral Perception and Autonomic Pattern Generation," Annual Review of Neuroscience, Vol. 25, 2002, pp. 433-469. doi:10.1146/annurev.neuro.25.032502.111311

[48] A. Raine, "Autonomic Nervous System Factors Underlying Disinhibited, Antisocial, and Violent Behavior. Biosocial Perspectives and Treatment Implications," Annals of the New York Academy of Sciences, Vol. 794, 1996, pp. 46-59. doi:10.1111/j.1749-6632.1996.tb32508.x
[49] C. J. Patrick, B. N. Cuthbert and P. J. Lang, "Emotion in the Criminal Psychopath: Fear Image Processing," Journal of Abnormal Psychology, Vol. 103, No. 3, 1994, pp. 523-534. doi:10.1037/0021-843X.103.3.523

[50] D. T. Lykken, "A Study of Anxiety in the Sociopathic Personality," Journal of Abnormal and Social Psychology, Vol. 55, No. 1, 1957, pp. 6-10. doi:10.1037/h0047232

[51] S. C. Herpertz, et al., "Emotion in Criminal Offenders with Psychopathy and Borderline Personality Disorder," Archives of General Psychiatry, Vol. 58, No. 8, 2001, pp. 737-745. doi:10.1001/archpsyc.58.8.737

[52] R. D. Hare, "Psychopathy, Fear Arousal and Anticipated Pain," Psychological Reports, Vol. 16, 1965, pp. 499502.

[53] D. M. P. Pavlovic and M. Aleksandra, "The Posterior Cingulate Cortex-From 'Laziness' to Complex Problem Solving," Curr Top Neurol Psychiatr Relat Discip, Vol. 18, No. 1, 2010.

[54] R. J. Maddock, "The Retrosplenial Cortex and Emotion: New Insights from Functional Neuroimaging of the Human Brain," Trends in Neurosciences, Vol. 22, No. 7, 1999, pp. 310-316. doi:10.1016/S0166-2236(98)01374-5

[55] R. J. Maddock, A. S. Garrett and M. H. Buonocore, "Posterior Cingulate Cortex Activation by Emotional Words: fMRI Evidence from a Valence Decision Task," Human Brain Mapping, Vol. 18, No. 1, 2003, pp. 30-41. doi:10.1002/hbm.10075

[56] G. Bush, P. Luu and M. I. Posner, "Cognitive and Emotional Influences in Anterior Cingulate Cortex," Trends in Cognitive Sciences, Vol. 4, No. 6, 2000, pp. 215-222. doi:10.1016/S1364-6613(00)01483-2

[57] P. Das, et al., "Pathways for Fear Perception: Modulation of Amygdala Activity by Thalamo-Cortical Systems," NeuroImage, Vol. 26, No. 1, 2005, pp. 141-148. doi:10.1016/j.neuroimage.2005.01.049

[58] N. Eisenberg, "Empathy-Related Responding and Prosocial Behaviour," Novartis Foundation Symposium, Vol. 278, 2007, pp. 71-80. 